\title{
The Utility of Keratin 903 as a New Prognostic Marker in Mass-Forming-Type Intrahepatic Cholangiocarcinoma
}

\author{
Shin-ichi Aishima, M.D., Yoshiki Asayama, M.D., Ken-ichi Taguchi, M.D., Keishi Sugimachi, M.D., \\ Ken Shirabe, M.D., Mitsuo Shimada, M.D., Keizo Sugimachi, M.D., Masazumi Tsuneyoshi, M.D. \\ Departments of Anatomic Pathology and of Surgery and Science, Graduate School of Medical Sciences, \\ Kyushu University, Fukuoka, Japan
}

The cytokeratins phenotype is largely preserved during neoplastic transformation and tumor development. We evaluated the immunoreactivity of biliary epithelial markers keratin 903 and cytokeratin 7 and 19 for intrahepatic cholangiocarcinoma, and compared the results with those for biliary dysplasia and hepatocellular carcinoma. Reactivity with keratin 903 was weakly expressed and increased after the expression of cytokeratin 7 and 19 during human intrahepatic bile duct development. More than $80 \%$ of cases of biliary dysplasia showed positive reactivity with keratin 903 . Of the 30 cases of hepatocellular carcinoma, $3(10 \%), 6(20 \%)$, and 1 (3\%) showed positive reactivity with Keratin 903 and cytokeratin 7 and 19, respectively. Among the 73 cases of intrahepatic cholangiocarcinoma, 54 (74\%), 66 $(90 \%)$, and $61(84 \%)$ showed positive reactivity with keratin 903 and cytokeratin 7 and 19, respectively. On clinicopathologic examination of intrahepatic cholangiocarcinomas, reduced keratin 903 reactivity was significantly higher in tumors with an expansive growth pattern $(P<.0001)$, in those with medullary-type stromal reaction $(P=.0327)$, in those without perineural invasion $(P=.0001)$, and in those without lymph node metastasis $(P=.0015)$. In addition, the reactivity with Keratin 903 was directly correlated with expression of cytokeratin 7 and $19(P=.0153$ and $P<.0001$, respectively). Cases showing reduced keratin 903 reactivity were characterized by a distinctive morphology indicating an hepatocellular carcinoma-like pattern. Multivariate analysis of overall survival revealed that keratin 903 reactivity was a significantly independent prognostic factor. In conclusion, patients with

Copyright (C) 2002 by The United States and Canadian Academy of Pathology, Inc.

VOL. 15, NO. 11, P. 1181, 2002 Printed in the U.S.A.

Date of acceptance: July 20, 2002.

Address reprint requests to: Masazumi Tsuneyoshi, M.D., Department of Anatomic Pathology, Graduate School of Medical Sciences, Kyushu University, 3-1-1 Maidashi, Higashi-ku, Fukuoka 812-8582, Japan; e-mail: masazumi@surgpath.med.kyushu-u.ac.jp; fax: 81-92-642-5968.

DOI: 10.1097/01.MP.0000032537.82380.69 intrahepatic cholangiocarcinoma showing reduced keratin 903 reactivity had a favorable prognosis. Remarkably, the cytokeratin phenotype of intrahepatic cholangiocarcinoma was correlated with the morphologic appearance of intrahepatic cholangiocarcinoma.

KEY WORDS: Intrahepatic cholangiocarcinoma, Keratin 903, Prognostic marker.

Mod Pathol 2002;15(11):1181-1190

The cytoskeleton is composed of microtubules, actin filaments, and intermediate filaments. Cytokeratins, which are the intermediate filaments in epithelial cells, are expressed in a cell-specific pattern according to various epithelial cell types (1). This cell-type specificity of cytokeratins is largely preserved during neoplastic transformation and tumor development (1). Numerous analyses of cytokeratins in primary liver tumor have shown that hepatocytes and the bile duct epithelium retain their distinct cytokeratin phenotype during neoplastic transformation (2-6). The classification of liver tumors by specific cytokeratin has thus been useful in clinical histodiagnosis (2, 3, 7-9).

Bile duct epithelia of various sizes in the liver and its neoplastic tumor, cholangiocarcinoma, have been shown to express large amounts of cytokeratins, including cytokeratins $7,8,18$, and 19 (1-3, $6-10)$. In addition, Gown and Vogel $(11,12)$ reported that a limited number of normal bile duct epithelia and cholangiocarcinomas stained positive with anti-cytokeratin $34 \beta \mathrm{E} 12$, whereas hepatocytes and hepatocellular carcinomas stained negative. The anti-cytokeratin antibody, $34 \beta \mathrm{E} 12$, which corresponds to keratin 903, is specific for high molecular weight cytokeratins (cytokeratin 1, 5, 10, and 14) as catalogued by Moll et al. (1). The antibody reacts with normal squamous and ductal epithelia as well as with carcinomas such as squamous cell carcinoma and adenocarcinoma of the breast, lung, and pancreas $(11,12)$. However, neither the reactivity for keratin 903 during human intrahepatic 
bile duct development nor that during neoplastic transformation of the bile duct epithelium has been adequately clarified.

Although the cytokeratin phenotype has been shown to be conserved during malignant transformation, the expression of biliary-typed cytokeratins-such as cytokeratin 7 and/or 19-has been observed in some hepatocellular carcinomas $(6,7$, 13-15). On the other hand, a small number of cholangiocarcinomas have shown weak expression of cytokeratin 7 and/or $19(7-9,15)$. To clarify the utility of cytokeratins known as biliary markers, we evaluated the reactivity with keratin 903 and expression of cytokeratin 7 and 19 in a large series of mass-forming-type intrahepatic cholangiocarcinomas with respect to clinicopathological prognostic factors and compared the results with those for biliary dysplasia and hepatocellular carcinoma, which are often difficult to differentiate from intrahepatic cholangiocarcinomas.

\section{MATERIALS AND METHODS}

\section{Tissue Specimens}

We examined 103 surgically resected liver tumors, consisting of 73 cases of intrahepatic cholangiocarcinoma and 30 cases of hepatocellular carcinoma, and 23 cases of hepatolithiasis with biliary dysplasia. We also evaluated fetal and infant liver specimens derived from autopsy (within $24 \mathrm{~h}$ ), including 18 fetal livers (gestational age range, 16 to $38 \mathrm{wk}$ ) and 5 infant livers (age range, $7 \mathrm{~d}$ to $3 \mathrm{y}$ ). These specimens were obtained by the Department of Anatomic Pathology of Kyushu University between July 1987 and May 2000. All the resected liver specimens were fixed in $10 \%$ formalin, embedded in paraffin, and stained with hematoxylin and eosin for histological examination. The diagnoses of biliary dysplasia, hepatocellular carcinoma, and intrahepatic cholangiocarcinoma were based on clinical information and made by routine histological examination. Of the 73 cases of intrahepatic cholangiocarcinoma, 10 were associated with other carcinomas, including stomach $(n=7)$ and colorectum $(n=3)$. Although, these cases were clinically suspected metastatic liver tumor, they were considered to be primary ICC, by morphological features and immunohistochemical technique using cytokeratin 7, 19, and 20 antibodies (7). Patients with intrahepatic cholangiocarcinoma who received nonradical surgery and died within 30 days after their operation were excluded.

\section{Macroscopic and Histologic Classification of Intrahepatic Cholangiocarcinoma}

Based on the definition by the Japanese Liver Cancer Study Group (16), intrahepatic cholangio- carcinoma was defined as a malignant tumor arising from the intrahepatic bile ducts and did not include tumors arising from the bile duct confluence and extrahepatic bile duct. All tumor s were grossly and histologically divided into subgroups. According to their macroscopic features, tumors were classified into the following three types: massforming, periductal-infiltrating, and intraductalgrowth type. In the current study, we examined mass-forming-type intrahepatic cholangiocarcinomas because a large percentage of the intrahepatic cholangiocarcinomas examined were of this type. Tumors were classified as peripheral $(n=54)$ or hilar ( $n=19)$ according to their location in the biliary tree. Intrahepatic cholangiocarcinomas were also histologically divided into the following four groups according to their degree of papillary or tubular formation: papillary adenocarcinoma and well-differentiated (15), moderately differentiated $(n=29)$, and poorly differentiated adenocarcinoma ( $n=29$ ). If more than one type was found, the predominant type was recorded, in order of the degree of involvement. Four cases of intrahepatic cholangiocarcinoma with an adenosquamous component were excluded because adenosquamous carcinoma of the liver, which shows strong reactivity with keratin 903, has been correlated with poor prognosis (17). We further categorized tumors into three types based on the degree of stromal reaction as described in a previous study (18). Areas in which the largest cut surface revealed a larger volume of fibrous stroma than tumor cells were defined as scirrhous areas. Tumors having a scirrhous area of $<30 \%$, that is, those with a prominent cellular component in a medullary growth and a faint stroma, were defined as medullary-type tumors ( $n$ $=25$ ). Tumors having a scirrhous area of $>70 \%$, that is, those with a prominent stromal desmoplasia and faint tumor cells, were defined as scirrhoustype tumors $(n=18)$. Tumors intermediate between medullary-type and scirrhous-type, that is, those having a scirrhous area between 30 and $70 \%$, were defined as intermediate-type tumors $(n=30)$. Tumors were also classified by growth pattern, with an infiltrating growth pattern defined by sinusoidal invasion of carcinoma cells at the border of the tumor, and an expansive growth pattern defined by invasion with compression of the surrounding liver parenchyma.

\section{Biliary Dysplasia and Hepatocellular Carcinoma Specimens}

The cases of hepatolithiasis with biliary dysplasia showed varying degrees of hyperplasia and/or dysplasia of the bile duct epithelium. Biliary dysplasia was defined as atypical bile duct with micropapillary proliferation into the ductal lumens. Dysplastic 
epithelial cells showed an increased nucleocytoplasmic ratio, loss of nuclear polarity and nuclear hyperchromasia. In this study, we especially evaluated dysplastic epithelium of the hepatolithiasis as a possible premalignant lesion.

Hepatocellular carcinomas with typical trabecular, pseudoglandular, and compact pattern were selected based on the staining of sections with hematoxylin and eosin. Histologically, hepatocellular carcinomas were classified as well differentiated ( $n$ $=5)$, moderately differentiated $(n=16)$ or poorly differentiated $(n=9)$.

\section{Immunohistochemical Staining}

In selecting appropriate sections for immunohistochemical examination, we favored those that included noncancerous liver tissue. When morphologic features of the tumor were heterogenous, sections from different areas were also examined. Immunohistochemical staining was carried out on serially cut paraffin sections using three monoclonal antibodies (keratin 903, cytokeratin 7 and 19). Four-micrometer sections of $10 \%$ formalin-fixed, paraffin-embedded tissues were deparaffinized in xylene and rehydrated in ethanol. The sections were digested with $0.1 \%$ trypsin solution at $37^{\circ} \mathrm{C}$ for 30 minutes. After the endogenous peroxidase activity was blocked by methanol containing $0.3 \%$ hydrogen peroxidase for 30 minutes, the slides were exposed to $10 \%$ nonimmunized rabbit serum in phosphate-buffered saline (PBS) for 10 minutes. Then the sections were incubated overnight at $4^{\circ} \mathrm{C}$ with primary antibodies. The primary antibodies used in this study were keratin 903 (34 $\beta$ E12, Enzo Diagnostics, New York) and cytokeratin 7 and 19 (DAKO, Glostrup, Denmark); all antibodies were used at a dilution of 1:50. Subsequent reaction was performed by the peroxidase-labeled streptavidinbiotin technique using a Histofine SAB-PO kit (Nichirei, Tokyo, Japan). Positive reaction was visualized with $\mathrm{H}_{2} \mathrm{O}_{2}$ containing 3,3'-diaminobenzidine tetrahydrochloride as chromogen, and the sections were counterstained with hematoxylin. Negative controls were established by substituting mouse normal serum for the primary antibodies.

\section{Quantitative Analysis}

For the staining of fetal and infant liver, the reactivity of immunostaining was classified using three scores based on the percentage and intensity of positive cells of bile ducts and ductal plates: specimens were considered negative $(-)$ when no cells were positive, focal and/or weak $(+)$ when $<50 \%$ of bile duct cells were positive and/or when the staining was weak compared with that of adult bile ducts, and strong $(++)$ when $>50 \%$ of cells were positive.

The immunostaining for biliary dysplasia and liver tumors was semiquantitatively defined using four scores based on the percentage of positive dysplastic epithelial and tumor cells: specimens were considered negative $(-)$ when no cells were positive, focal $(1+)$ when $<10 \%$ of cells were positive, moderate $(2+)$ when 10 to $50 \%$ of cells were positive, and diffuse $(3+)$ when $>50 \%$ of cells were positive. These scores were divided into two groups: $2+/ 3+$ cases were considered to show preserved reactivity, and $0 / 1+$ cases, to show reduced reactivity.

The cases showing reduced cytokeratin 7 or 19 or keratin 903 reactivity were immunostained by antihepatocyte antigen (HepPar 1, OCHIE5, DAKO). HepPar 1 antibody is a useful marker for the differential diagnosis of hepatocellular carcinoma (19). For staining of HepPar 1, sections were treated with sodium citrate buffer ( $\mathrm{pH}$ 6.0) for 15 minutes at $99^{\circ}$ $\mathrm{C}$ in a microwave oven. Subsequent reaction was performed as same as cytokeratin staining.

\section{Statistical Analysis}

The correlation between the reactivity of Keratin 903 and clinicopathologic factors was assessed by means of the $\chi^{2}$ test, Mann-Whitney $U$ test, and Fisher's exact test. The adjusted survival time was taken as the period of survival between surgery and the date of the last follow-up or death by disease. Overall survival curves were constructed by the Kaplan-Meier method and statistically analyzed with the Logrank (Mantel-Cox) test. Cox's proportional hazards model with a stepwise procedure was used in the multivariate survival analysis. $P$ values of $<.05$ were considered to indicate statistical significance.

\section{RESULTS}

\section{Fetal and Infant Liver}

The reactivity of cytokeratins during intrahepatic bile duct development is summarized in Table 1 . In fetal and infant liver $(n=23)$, cytokeratin 19 was diffusely expressed in all the immature bile duct cells and the cells of the ductal plate, which were in direct contact with the mesenchyma around the portal vein and the branches of portal veins after 16 weeks of gestation, and this expression persisted in more mature bile duct cells and bile ductules (Fig. 1A, B). In contrast, cytokeratin 7 was expressed weakly in approximately half of the immature bile duct epithelial cells and focally in the cells of the ductal plate until 21 weeks of gestation (Fig. 1C). As bile duct differentiation progressed, cytokeratin 7 
TABLE 1. The Reactivity of Keratin 903, Cytokeratin 7 (CK 7), and Cytokeratin 19 (CK 19) in Fetal and Newborn Liver

\begin{tabular}{lcccc}
\hline \multicolumn{1}{c}{ Age } & $\begin{array}{c}\text { No. of cases } \\
(N=23)\end{array}$ & Keratin 903 & CK 7 & CK 19 \\
\hline Gestational (weeks) & & & & \\
16 & 4 & - & - & ++ \\
19 & 2 & - & + & ++ \\
21 & 1 & - & + & ++ \\
23 & 2 & - & + & ++ \\
28 & 2 & - & + & ++ \\
31 & 1 & + & ++ & ++ \\
33 & 3 & + & ++ & ++ \\
35 & 2 & + & ++ & ++ \\
38 & 1 & & & ++ \\
After birth & & + & ++ & ++ \\
$1 / 2$ mo & 1 & + & ++ & ++ \\
1 mo & 1 & ++ & ++ & ++ \\
4 mo & 1 & ++ & ++ & ++ \\
2 y & 1 & ++ & ++ & ++ \\
3 y & 1 & & & ++ \\
\hline
\end{tabular}

expression increased and persisted in bile duct epithelial cells (Fig. 1D). Keratin 903 reactivity was found weakly in bile duct cells starting at 33 weeks of gestation, but the intensity of staining was low in the fetal liver (Fig. 1E, F). The intensity of keratin 903 reactivity was equal to that of cytokeratin 7 and 19 after birth.

\section{Biliary Dysplasia of the Liver}

All normal biliary tracts of the human adult liver, including large, septal, and interlobular bile ducts and bile ductules, were extensively positive for keratin 903 and cytokeratin 7 and 19, whereas hepatocytes were negative for keratin 903 and cytokeratins 7 and 19. All 23 cases with biliary dysplasia showed preserved expression of cytokeratin 7 and 19 (Fig. $2 \mathrm{~A})$, whereas 4 cases (17\%) showed negative or focal staining for keratin 903 (Fig. 2B; Table 2).

\section{Hepatocellular Carcinoma}

Among the 30 cases of hepatocellular carcinoma, $2(7 \%)$ and $1(3 \%)$ showed moderate staining for keratin 903 and cytokeratin 19, respectively. More than $90 \%$ of hepatocellular carcinomas showed negative or focal staining for keratin 903 and cytokeratin 19 (Fig. 2C). In contrast, 6 (20\%) of the cases of hepatocellular carcinoma showed moderate to diffuse staining for cytokeratin 7 (Fig. 2D). Among cytokeratin 7-positive hepatocellular carcinomas, three were moderately differentiated, and two, welldifferentiated, and all five showed a thin trabecular, acinar, and pseudoglandular pattern.

\section{Cholangiocarcinoma}

Positive staining for keratin 903 and cytokeratin 7 and 19 was seen in the cytoplasm and membrane. Of the 73 cases, 54 (74\%) showed preserved reactivity with keratin 903 (Fig. 2E). The remaining 19 cases with reduced reactivity were composed of 15 negative and 4 focal staining for keratin 903. Cytokeratin 7 was strongly expressed in 66 cases $(90 \%)$, whereas only 3 showed negative, and 4, focal staining, for cytokeratin 7. Cytokeratin 19 was strongly expressed in 61 cases (84\%), whereas 8 showed negative, and 4, focal staining, for cytokeratin 19 (Table 2).

Clinical and gross findings including age, sex, tumor location, and tumor size were not correlated with keratin 903 reactivity. Analysis of histologic findings of intrahepatic cholangiocarcinomas showed that reduced keratin 903 reactivity was significantly higher in tumors with an expansive growth pattern $(P<.0001)$, those with medullary type stromal reaction $(P=.0327)$, those without perineural invasion $(P=.0001)$ and those without lymph node metastasis $(P=.0015)$, but was not correlated with venous invasion or intrahepatic metastasis (Table 3). Intrahepatic cholangiocarcinomas showing reduced keratin 903 reactivity had an expansive growth pattern and a faint stromal reaction at the marginal portion of the tumor. Characteristically, these intrahepatic cholangiocarcinomas frequently showed a proliferation of compact sheets and solid nests of tumor cells with scanty fibrous stroma, similar to the appearance of hepatocellular carcinoma (Fig. 3A-B). However, mucin production was observed in these tumor cells by staining with Alcian blue (Fig. 3D). The tumors with reduced cytokeratin 7 and 19 or keratin 903 reactivity revealed no staining for HepPar 1 (Fig. 3C). Interestingly, in the limited areas in which the tumor cells showed a proliferation of sheets and nests, staining for keratin 903 was negative or very weak, even in the strong keratin 903-reactive cases. With regard to the correlation among the reactivity of keratin 903 and cytokeratin 7 and 19, keratin 903 reactivity was correlated with cytokeratin 7 expression and cytokeratin 19 expression $(P=.0153$ and $P<.0001$, respectively; Table 3 ). Clinicopathological analysis for cytokeratin 7 and 19 revealed no correlation between these markers and any of the parameters examined.

\section{Prognosis}

The median follow-up period for the patients with intrahepatic cholangiocarcinoma was 2.2 years (maximum, $10 \mathrm{y}$ ). The patients with intrahepatic cholangiocarcinoma who received nonradical surgery and died within 30 days after the operation were excluded. The patients with intrahepatic cholangiocarcinoma characterized by reduced keratin 903 reactivity showed a significantly more favorable overall survival rate than those with preserved keratin 903 reactivity ( $P=.0015$; Fig. 4$)$. Further evaluation by multivariate analysis using Cox's propor- 


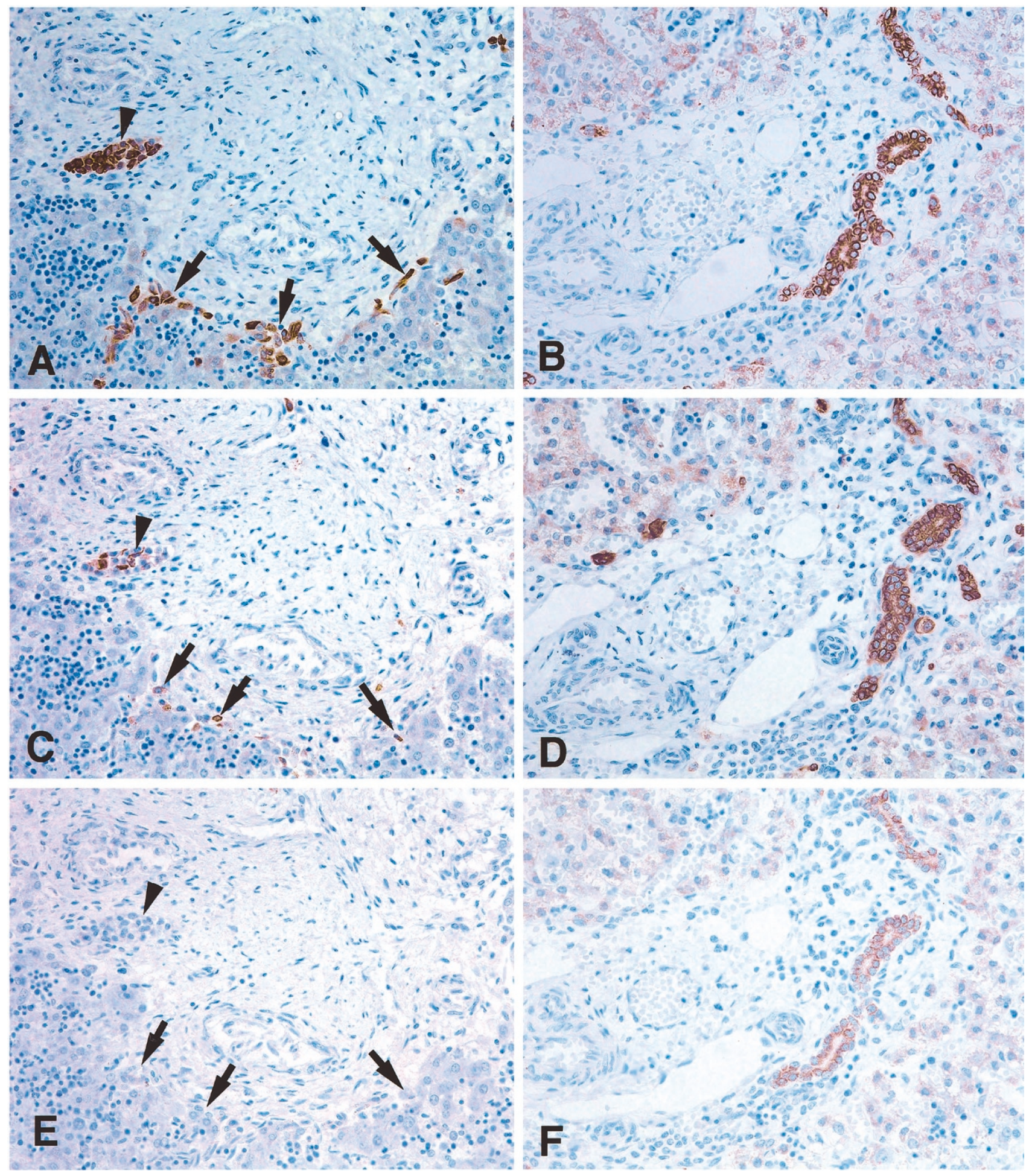

FIGURE 1. Human fetal liver, 16 weeks of gestation: part of the portal tract and adjacent liver parenchyma are shown; the immature bile duct cells (arrowhead) and the cells of the ductal plate (arrows) were strongly positive for cytokeratin 19 (A), and in contrast, these cells were weakly and focally positive for cytokeratin 7 (C); note the absence of keratin 903 staining in these cells (E). Human fetal liver, 35 weeks of gestation: the bile ducts and ductules were strongly positive for cytokeratin 19 and 7 (B, D) but weakly positive for keratin 903 (F). The liver parenchymal cells were negative for all cytokeratins.

tional hazards model revealed that the presence of intrahepatic metastasis, lymphatic permeation, keratin 903 reactivity, and histologic differentiation were independent prognostic factors (Table 4).

\section{DISCUSSION}

The intrahepatic bile ducts develop from the primitive hepatocytes that surround the branches of the 

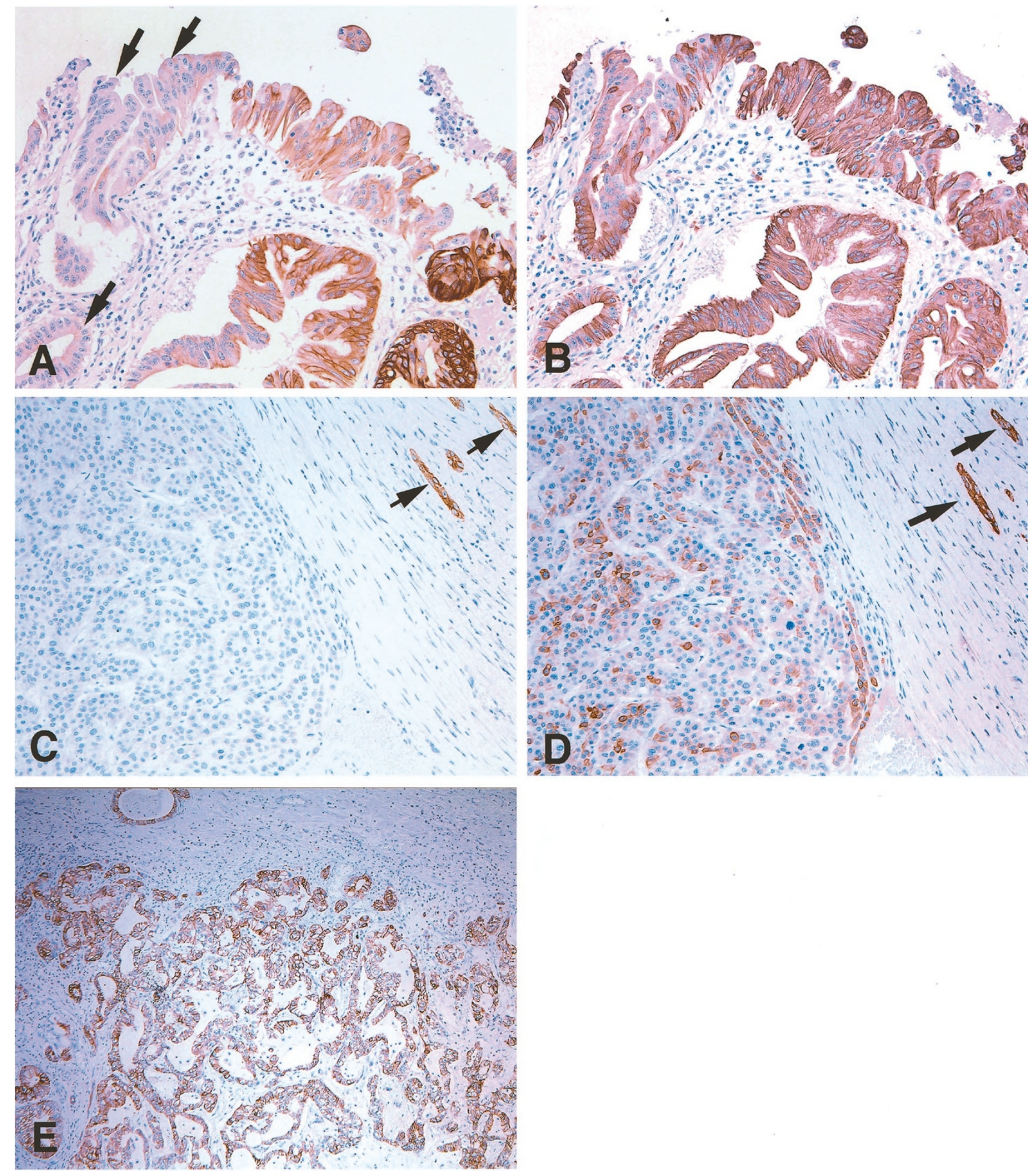

FIGURE 2. Biliary dysplasia with hepatolithiasis. Micropapillary projection with cellular atypia into the bile duct lumen was evident. One half of the dysplastic epithelium (arrows) was weak or negative for Keratin 903 (A), whereas these cells were strongly positive for cytokeratin 7 (B). Moderately differentiated hepatocellular carcinoma growing a trabecular pattern was negative for keratin 903 (C) but showed strong reactivity for cytokeratin 7 (D). The bile ducts in noncancerous area were positive for keratin 903 and cytokeratin 7 (arrows). Poorly differentiated adenocarcinoma proliferated in a microglandular fashion, invading the surrounding liver parenchyma. The carcinoma cells were diffusely positive for keratin $903(\mathbf{E})$.

portal vein, as revealed by immunohistochemical analyses of cytokeratin expression. And although the expressions of cytokeratin 7 and 19 during intrahepatic bile duct development have been fully investi- gated, reactivity with keratin 903 has rarely been studied (20-22). In the present study, we demonstrated that keratin 903 reactivity was weak compared with that with cytokeratin 7 and 19 at the gestational stage 
TABLE 2. The Reactivity of Keratin 903, CK 7, and CK 19 in Dysplasia and Liver Tumors

\begin{tabular}{lccc}
\hline \multicolumn{1}{c}{ Marker } & $\begin{array}{c}\text { Dysplasia } \\
(N=23)\end{array}$ & $\begin{array}{c}\text { HCC } \\
(N=30)\end{array}$ & $\begin{array}{c}\text { ICC } \\
(N=73)\end{array}$ \\
\hline Keratin 903 & & & \\
- & 0 & 27 & 15 \\
$1+(n)$ & 4 & 1 & 4 \\
$2+(n)$ & 8 & 2 & 15 \\
$3+(n)$ & 11 & 0 & 39 \\
$\quad$ Preserved reactivity, $n(\%)$ & $19(83)$ & $2(7)$ & $54(74)$ \\
CK 7 & & & \\
$-(n)$ & 0 & 19 & 3 \\
$1+(n)$ & 0 & 5 & 4 \\
$2+(n)$ & 0 & 5 & 13 \\
$3+(n)$ & 23 & 1 & 53 \\
Preserved expression, $n$ (\%) & $23(100)$ & $6(20)$ & $66(90)$ \\
CK 19 & & & \\
- & 0 & 29 & 8 \\
$1+$ & 0 & 0 & 4 \\
$2+$ & 2 & 1 & 16 \\
$3+$ & 21 & 0 & 45 \\
Preserved expression & $23(100)$ & $1(3)$ & $61(84)$ \\
\hline
\end{tabular}

CK 7, cytokeratin 7; CK 19, cytokeratin 19; HCC, hepatocellular carcinoma; ICC, intrahepatic cholangiocarcinoma.

and that keratin 903 reactivity increased gradually after birth. These results suggest that keratin 903 is as effective as cytokeratin 7 and 19 as a marker of the mature bile duct epithelium. It may be possible that keratin 903 reactivity was related to the higher maturation of the bile duct epithelium in this study because keratin 903 reactivity was detected at the late stage of intrahepatic bile duct development.

Normal human hepatocytes have been shown to express cytokeratin 8 and 18, whereas normal bile duct cells in the liver express cytokeratin $7,8,18$, and $19(1,10)$. This cytokeratin pattern is considered to be largely conserved in malignant tumors of the liver (2-5), but a small number of hepatocellular carcinomas have also been characterized by an unexpected bile duct-type cytokeratin pattern $(6,7$, 13-15). Several investigators have already pointed out that some hepatocellular carcinomas contain tumor cells positive for cytokeratin 7 and/or 19, indicating that synthesis of bile duct-type cytokeratin not normally present in hepatocytes can take place $(6,10,14)$. Our present results also indicated cytokeratin 7 expression in some hepatocellular carcinomas. Thus the cytokeratin pattern might not always be preserved during malignant transformation of the liver. Some studies, including our previous one, have reported that $>90 \%$ of cholangiocarcinomas showed positivity for cytokeratin 7 or 19 (7-9). The cytokeratin reactivity in these studies was classified into four scores, and positive cases included those of focal staining in which $<10 \%$ of tumor cells were positive. In the current study, to emphasize the focal or negative staining, we defined cases of reduced reactivity as those in which $<10 \%$ of tumor cells were positive. According to this definition, $26 \%, 10 \%$, and $16 \%$ of cases showed reduced reactivity for keratin 903 and
TABLE 3. Clinicopathological Findings between Preserved and Reduced Keratin 903 Reactivity in ICCs

\begin{tabular}{|c|c|c|c|}
\hline Variable & $\begin{array}{c}\text { Preserved } \\
(n=54)\end{array}$ & $\begin{array}{l}\text { Reduced } \\
(n=19)\end{array}$ & $P$ Value \\
\hline Age $($ mean $\pm S D, y)$ & $64.2 \pm 9.6$ & $59.4 \pm 12.0$ & 0.1201 \\
\hline Sex & & & 0.9791 \\
\hline Male & 32 & 12 & \\
\hline Female & 22 & 7 & \\
\hline Location & & & 0.2832 \\
\hline Hilar type & 14 & 2 & \\
\hline Peripheral type & 40 & 17 & \\
\hline Size (cm) & & & 0.3185 \\
\hline$>4.5$ & 29 & 7 & \\
\hline$<4.5$ & 25 & 12 & \\
\hline Histological differentiation & & & 0.6435 \\
\hline Papillary and well & 10 & 5 & \\
\hline Moderately & 21 & 8 & \\
\hline Poorly & 23 & 6 & \\
\hline Growth pattern & & & $<0.0001^{*}$ \\
\hline Expansive & 10 & 14 & \\
\hline Infiltrating & 44 & 5 & \\
\hline Stromal reaction & & & $0.0327^{*}$ \\
\hline Medullary & 14 & 11 & \\
\hline Intermediate & 24 & 6 & \\
\hline Scirrhous & 16 & 2 & \\
\hline Vascular invasion & & & 0.3344 \\
\hline Positive & 37 & 10 & \\
\hline Negative & 17 & 9 & \\
\hline Lymphatic permeation & & & 0.0550 \\
\hline Positive & 38 & 8 & \\
\hline Negative & 16 & 11 & \\
\hline Perineural invasion & & & $0.0001^{*}$ \\
\hline Positive & 41 & 4 & \\
\hline Negative & 13 & 15 & \\
\hline Intrahepatic metastasis & & & 0.1635 \\
\hline Positive & 29 & 6 & \\
\hline Negative & 25 & 13 & \\
\hline Lymph node metastasis & & & $0.0015^{*}$ \\
\hline Positive & 27 & 1 & \\
\hline Negative & 27 & 18 & \\
\hline CK 7 expression & & & $0.0153^{*}$ \\
\hline Preserved & 52 & 14 & \\
\hline Reduced & 2 & 5 & \\
\hline CK 19 expression & & & $<0.0001^{*}$ \\
\hline Preserved & 52 & 9 & \\
\hline Reduced & 2 & 10 & \\
\hline
\end{tabular}

ICC, intrahepatic cholangiocarcinoma

${ }^{*} P<.05$.

cytokeratin 7 and 19, respectively. These results suggest that expression of bile duct-type cytokeratin was reduced or lost in a small number of cholangiocarcinoma during neoplastic transformation or tumor development.

Before this study, the character of cholangiocarcinoma with phenotypic change of cytokeratin was unclear. We demonstrated that $26 \%$ of intrahepatic cholangiocarcinomas showed negative or focal staining for keratin 903 . We also found that intrahepatic cholangiocarcinomas with reduced keratin 903 reactivity showed distinctive morphological features, that is, an expansive growth pattern with scanty stromal reaction. Histologically, the tumor cells with reduced keratin 903 reactivity proliferated in sheets and nests, which was similar to the trabecular pattern of hepatocellular carcinoma. Several studies have discussed the cases of cholangiocarcinoma with hepatocellular carcinoma-like 

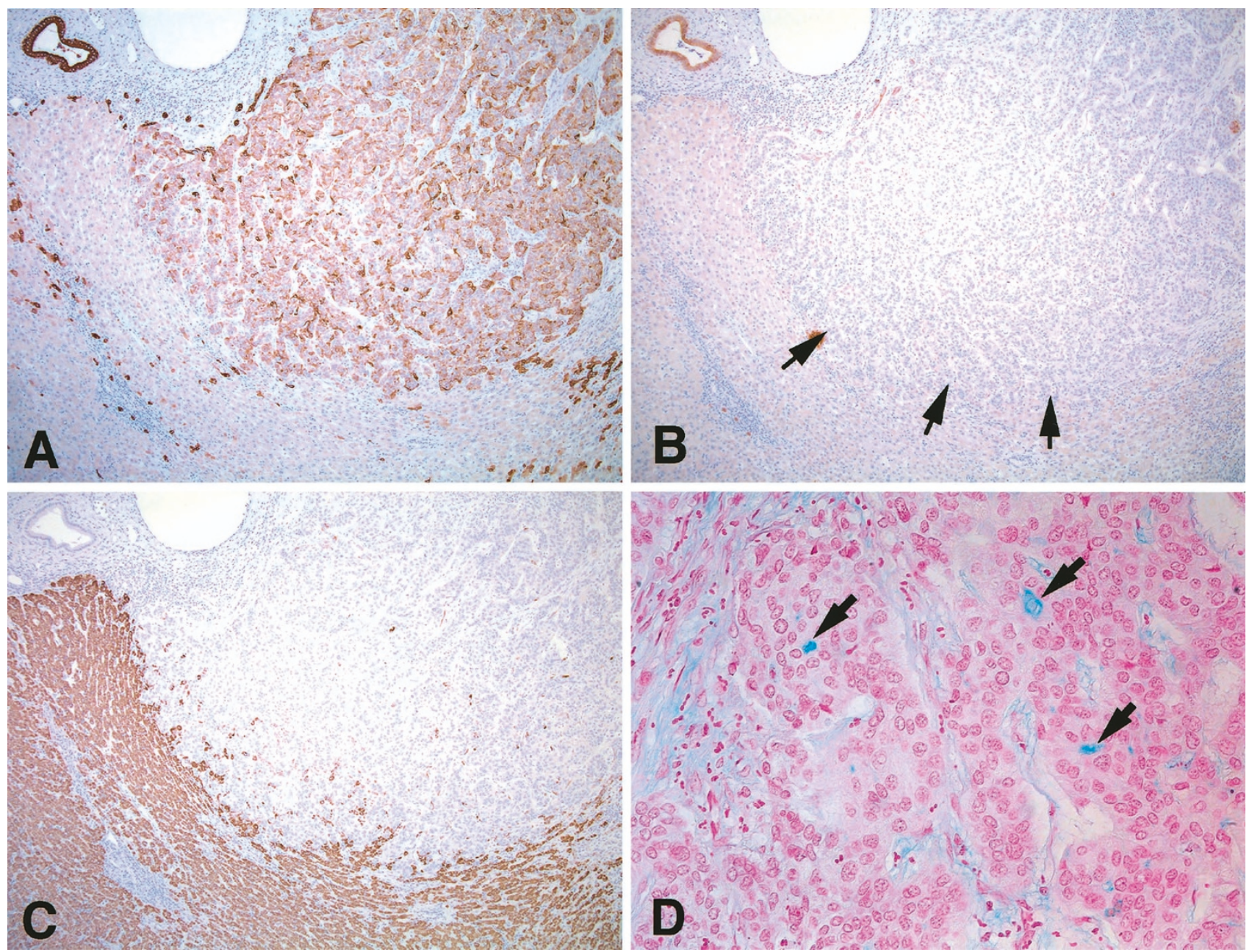

FIGURE 3. Mass-forming-type intrahepatic cholangiocarcinoma. Carcinoma cells proliferating in sheets and nests compressed the noncancerous liver tissue (arrows). The carcinoma cells were diffusely positive for cytokeratin 7 (A) but negative for keratin 903 (B). The noncancerous liver parenchyma was strongly positive for HepPar 1, but the carcinoma cells were negative for HepPar 1 (C). Mucin production (arrows) was observed in these tumor cells by staining with Alcian blue (D). The bile duct epithelium was positive for keratin 903 and cytokeratin 7 and 19 .

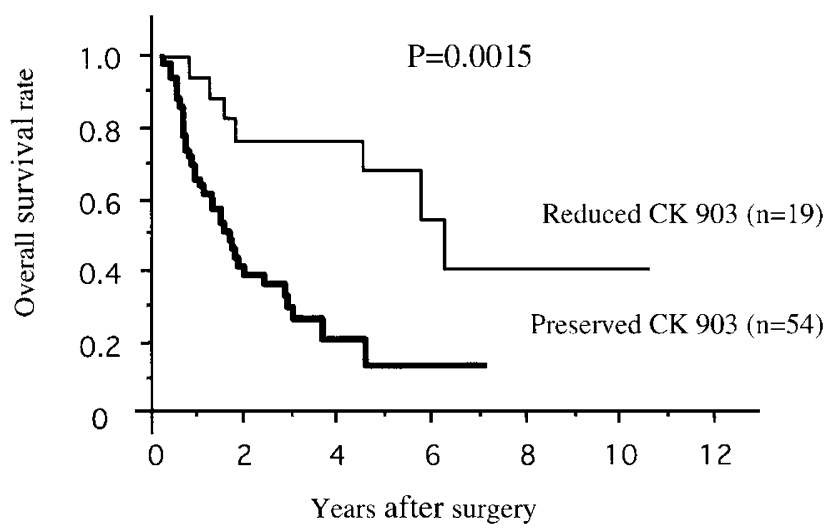

FIGURE 4. Those patients with intrahepatic cholangiocarcinoma characterized by reduced keratin 903 reactivity $(n=19)$ showed a significantly more favorable overall survival rate than did those with preserved keratin 903 reactivity $(n=54)$.

features. Yamamoto et al. (23) reported a case of small nodular-type cholangiocarcinoma showing similarities to hepatocellular carcinoma. Okuda et
TABLE 4. Stepwise Multivariate Survival Analysis with Cox's Proportional Hazards Model

\begin{tabular}{lcccc}
\hline \multicolumn{1}{c}{ Variable } & Coefficient & $\begin{array}{c}\text { Coefficient/ } \\
\text { S.E. }\end{array}$ & $\begin{array}{c}\text { Hazards } \\
\text { Ratio }\end{array}$ & $P$ Value \\
\hline Intrahepatic metastasis & 1.5234 & 4.1559 & 4.5880 & 0.0001 \\
Lymphatic permeation & 1.4676 & 3.4250 & 4.3389 & 0.0002 \\
Keratin 903 reactivity & 1.3705 & 2.9088 & 3.9372 & 0.0010 \\
Histologic differentiation & 0.9560 & 2.7493 & 0.3844 & 0.0060 \\
\hline
\end{tabular}

S.E., standard error.

al. (24) reported that peripheral-type intrahepatic cholangiocarcinoma behaves in a manner intermediate between those of hilar bile duct carcinoma and hepatocellular carcinoma. Yamanaka et al. (25) have noted that mass-forming-type intrahepatic cholangiocarcinomas without biliary stricture behave more like hepatocellular carcinomas. Generally, intrahepatic cholangiocarcinomas show a tendency to invade the lymphatic channel and metastasize to lymph nodes and remote organs, whereas hepatocellular carcinomas show a tendency of venous invasion via the portal and hepatic 
veins and intrahepatic metastasis. Our histologic analysis showed that the cases with reduced keratin 903 reactivity showed less invasive to the lymph nodes but had a tendency of venous invasion and intrahepatic metastasis. Our results suggest that the cases with reduced keratin 903 reactivity had a biologic behavior similar to hepatocellular carcinoma. However, it is important to differentiate the tumors with reduced keratin 903 reactivity from the hepatocellular carcinoma or the combined hepatocellular carcinoma and cholangiocarcinoma. In the components of reduced keratin 903 reactivity, we observed three conclusive pieces of evidence for our suggestion: (1) mucin production and glandular components were clearly observed; (2) bile production, sinusoidal structure, and Mallory body formation were absent; and (3) HepPar 1 staining was immunohistochemically absent. For differentiating cholangiocarcinoma from hepatocellular carcinoma, there are excellent markers such as HepPar 1 now (19). Therefore, what we have called intrahepatic cholangiocarcinoma with reduced keratin 903 reactivity is, in this author's opinion, intrahepatic cholangiocarcinoma with hepatocellular carcinoma-like features, not combined hepatocellular carcinoma and cholangiocarcinoma. HepPar 1 staining would prove that.

Our multivariate analysis showed that reduced keratin 903 reactivity is a new and more effective independent prognostic factor. We showed that patients of intrahepatic cholangiocarcinoma with reduced keratin 903 reactivity were more likely to have less-invasive carcinoma. We previously demonstrated other prognostic factors, such as p27 and cyclin D1 $(26,27)$. The current study is the first to demonstrate that reduced keratin 903 reactivity is a marker of favorable prognosis in intrahepatic cholangiocarcinomas.

We also examined the cytokeratin expression of cases with intrahepatic biliary dysplasia. Biliary dysplasia is considered the premalignant condition for cholangiocarcinoma $(28,29)$. On microscopic examination, there is no histologic difference between the dysplastic epithelial cells showing weak reactivity of keratin 903 and those cells showing strong reactivity. However, the findings suggest that some biliary epithelial cells show the loss of the bile duct-type cytokeratin phenotype in the premalignant condition.

Previously, the mechanism by which the cytokeratin phenotype alters morphogenesis and tumor aggressiveness had been uncertain. However, altered cytokeratin expression has been shown to affect both cell morphology and invasive potential in squamous cell carcinomas (30). In epithelial cells, cytokeratins are arranged in a complex network of desmoplakin and extracellular matrix receptors such as integrins, suggesting that cytokera- tins may be involved in signal transduction from the extracellular matrix (31-33). The extracellular matrix composition may play a role in the phenotypic change of hepatocytes (34). The microscopic features of tumor specimens having reduced keratin 903 reactivity tended to show medullary growth with faint fibrous stroma. These results indicate that extracellular matrix composition and mesenchymal cells may play a role in the phenotypic change of bile-duct-type cytokeratin in intrahepatic cholangiocarcinoma.

Our study suggests that keratin 903 immunostaining may provide important prognostic information on patients with intrahepatic cholangiocarcinoma because Keratin 903 reactivity was shown to be a significantly independent prognostic factor. It must be stressed that the reduced Keratin 903 reactivity was associated with reduction in the aggressiveness of intrahepatic cholangiocarcinoma. Finally, the most surprising finding was that the cytokeratin phenotype of tumors was correlated with the morphologic appearance of intrahepatic cholangiocarcinoma.

\section{REFERENCES}

1. Moll R, Franke WW, Schiller DL, Geiger B, Krepler R. The catalog of human cytokeratins: patterns of expression in normal epithelia, tumors and cultured cells. Cell 1982;31:1124.

2. Balaton AJ, Nehama-Sibony M, Gotheil C, Callard P, Baviera EE. Distinction between hepatocellular carcinoma, cholangiocarcinoma, and metastatic carcinoma based on immunohistochemical staining for carcinoembryonic antigen and for cytokeratin 19 on paraffin sections. J Pathol 1988;156:305-10.

3. Johnson DE, Herndier BG, Medeiros LJ, Warnke RA, Rouse RV. The diagnostic utility of the keratin profiles of hepatocellular carcinoma and cholangiocarcinoma. Am J Surg Pathol 1988;12:187-97.

4. Denk H, Krepler R, Lackinger E, Artlieb U, Franke WW. Biochemical and immunocytochemical analysis of the intermediate filament cytoskeleton in human hepatocellular carcinomas and in hepatic neoplastic nodules of mice. Lab Invest 1982;46:584-96.

5. Lai YS, Thung SN, Gerber MA, Chen ML, Schaffner F. Expression of cytokeratins in normal and diseased livers and in primary liver carcinomas. Arch Pathol Lab Med 1989;113: 134-8.

6. Fischer HP, Altmannsberger M, Weber K, Osborn M. Keratin polypeptides in malignant epithelial liver tumors. Differential diagnostic and histogenetic aspects. Am J Pathol 1987; $127: 530-7$

7. Maeda T, Kajiyama K, Adachi E, Takenaka K, Sugimachi K, Tsuneyoshi M. The expression of cytokeratins 7, 19, and 20 in primary and metastatic carcinomas of the liver. Mod Pathol 1996;9:901-9.

8. Shimonishi T, Miyazaki K, Nakanuma Y. Cytokeratin profile relates to histological subtypes and intrahepatic cholangiocarcinioma and primary site of metastatic adenocarcinoma of liver. Histopathology 2000;37:55-63.

9. Rullier A, Le Bail B, Fawaz R, Blanc JF, Saric J, Bioulac-Sage P. Cytokeratin 7 and 20 expression in cholangiocarcinomas varies along the biliary tract but still differs from that in 
colorectal carcinoma metastasis. Am J Surg Pathol 2000;24: 870-6.

10. Van Eyken P, Desmet VJ. Cytokeratins and the liver. Liver 1993;13:113-22.

11. Gown AM, Vogel AM. Monoclonal antibodies to human intermediate filament proteins. II. Distribution of filament proteins in normal human tissues. Am J Pathol 1984;114: 309-21.

12. Gown AM, Vogel AM. Monoclonal antibodies to human intermediate filament proteins. III. Analysis of tumors. Am J Clin Pathol 1985;84:413-24.

13. Van Eyken P, Sciot R, Paterson A, Callea F, Kew MC, Desmet VJ. Cytokeratin expression in hepatocellular carcinoma: an immunohistochemical study. Hum Pathol 1988;19:562-8.

14. Hurlimann J, Gardiol D. Immunohistochemistry in the differential diagnosis of liver carcinomas. Am J Surg Pathol 1991;15:280-8.

15. D’Errico A, Baccarini P, Fiorentino M, Ceccarelli C, Bonazzi C, Ponzetto A, et al. Histogenesis of primary liver carcinomas: strengths and weaknesses of cytokeratin profile and albumin mRNA detection. Hum Pathol 1996;27:599-604.

16. Liver Cancer Study Group. The general rules for the clinical and pathological study of primary liver cancer. 4 th ed. Tokyo: Kanehara Publications; 2000.

17. Maeda T, Takenaka K, Taguchi K, Kajiyama K, Shirabe K, Shimada $\mathrm{M}$, et al. Adenosquamous carcinoma of the liver: clinicopathologic characteristics and cytokeratin profile. Cancer 1997;80:364-71.

18. Kajiyama K, Maeda T, Takenaka K, Sugimachi K, Tsuneyoshi M. The significance of stromal desmoplasia in intrahepatic cholangiocarcinoma: a special reference of "scirrhous-type" and "nonscirrhous-type" growth. Am J Surg Pathol 1999;23: 892-902.

19. Minervini MI, Demetris AJ, Lee RG, Carr BI, Madariaga J, Nalesnik MA. Utilization of hepatocyte-specific antibody in the immunocytochemical evaluation of liver tumors. Mod Pathol 1997;10:686-92.

20. Van Eyken P, Sciot R, Callea F, Van der Steen K, Moerman P, Desmet VJ. The development of the intrahepatic bile ducts in man: a keratin-immunohistochemical study. Hepatology 1988;8:1586-95.

21. Van Eyken P, Sciot R, Desmet V. Intrahepatic bile duct development in the rat: a cytokeratin-immunohistochemical study. Lab Invest 1988;59:52-9.

22. Shah KD, Gerber MA. Development of intrahepatic bile ducts in humans. Immunohistochemical study using mono- clonal cytokeratin antibodies. Arch Pathol Lab Med 1989; 113:1135-8.

23. Yamamoto M, Takasaki K, Nakano M, Saito A. Minute nodular intrahepatic cholangiocarcinoma. Cancer 1998;82:2145-9.

24. Okuda K, Kubo Y, Okazaki N, Arishima T, Hashimoto M. Clinical aspects of intrahepatic bile duct carcinoma including hilar carcinoma: a study of 57 autopsy-proven cases. Cancer 1977;39:232-46.

25. Yamanaka N, Okamoto E, Ando T, Oriyama T, Fujimoto J, Furukawa $\mathrm{K}$, et al. Clinicopathologic spectrum of resected extraductal mass-forming intrahepatic cholangiocarcinoma. Cancer 1995;76:2449-56.

26. Taguchi K, Aishima S, Asayama Y, Kajiyama K, Kinukawa N, Shimada M, et al. The role of p27kip1 protein expression on the biological behavior of intrahepatic cholangiocarcinoma. Hepatology 2001;33:1118-23.

27. Sugimachi K, Aishima S, Taguchi K, Tanaka S, Shimada M, Kajiyama $\mathrm{K}$, et al. The role of overexpression and gene amplification of cyclin D1 in intrahepatic cholangiocarcinoma. J Hepatol 2001;35:74-9.

28. Terada T, Nakanuma Y, Ohta T, Nagakawa T. Histological features and interphase nucleolar organizer regions in hyperplastic, dysplastic and neoplastic epithelium of intrahepatic bile ducts in hepatolithiasis. Histopathology 1992;21: 233-40.

29. Koga A, Ichimiya H, Yamaguchi K, Miyazaki K, Nakayama F. Hepatolithiasis associated with cholangiocarcinoma. Possible etiologic significance. Cancer 1985;55:2826-9.

30. Crowe DL, Milo GE, Shuler CF. Keratin 19 downregulation by oral squamous cell carcinoma lines increases invasive potential. J Dent Res 1999;78:1256-63.

31. Kouklis PD, Hutton E, Fuchs E. Making a connection: direct binding between keratin intermediate filaments and desmosomal proteins. J Cell Biol 1994;127:1049-60.

32. Bornslaeger EA, Corcoran CM, Stappenbeck TS, Green KJ. Breaking, the connection: displacement of the desmosomal plaque protein desmoplakin from cell-cell interfaces disrupts anchorage of intermediate filament bundles and alters intercellular junction assembly. J Cell Biol 1996;134:9851001.

33. Yancey KB. Adhesion molecules. II. Interactions of keratinocytes with epidermal basement membrane. J Invest Dermatol 1995;104:1008-14.

34. Matsumoto S, Yamamoto K, Nagano T, Okamoto R, Ibuki N, Tagashira M, et al. Immunohistochemical study on phenotypical changes of hepatocytes in liver disease with reference to extracellular matrix composition. Liver 1999;19:32-8. 